\title{
Intersection of biobanking and clinical care: should discrepant diagnoses and pathological findings be returned to research participants?
}

\author{
Nicole C. Lockhart, PhD ${ }^{1}$, Rihab Yassin, PhD², Carol J. Weil, JD ${ }^{1}$ and Carolyn C. Compton, MD, PhD ${ }^{1}$
}

Diagnostic discrepancies occur when the diagnosis made on a biospecimen during the course of review at a biobank differs from the original clinical diagnosis. These diagnostic discrepancies detected during biobanking present unique challenges that are distinct from other types of research results or incidental findings. The proposed process for reporting diagnostic discrepancies or pathological incidental findings identified through a quality assurance evaluation at the biobank includes verification of the biospecimen identity, verification of the diagnosis within the biobank, and re-review of the case by the pathologist at the biospecimen collection site. If the pathologist at the biobank and the original pathologist do not reach

Although significant attention has been given to the management of incidental findings ${ }^{1}$ and individual genetic results discovered in the course of research, ${ }^{2,3}$ human biospecimens collected for research present potential clinical management challenges and considerations, particularly in a research setting. If biospecimens are originally obtained for clinical purposes, human research participants may not have consented to use of their biospecimens and accordingly may be unaware that their biospecimens are being utilized in research. ${ }^{4}$ In other cases, the research participant may have consented to the use of his/her biospecimen in research, but the scope of that consent may be unclear or the consent may not have addressed the issue of return of research results or incidental findings. ${ }^{4}$ If biospecimens are collected for undefined future research, it may be challenging to describe possible research results or incidental findings in the informed consent document due to the range of possible findings. ${ }^{5}$

In addition to results that may be discovered in the course of research, the biobank may discover important information relevant to patient care. In the case of tissue biospecimens in particular, quality control measures or other pathological examinations performed by the biobank may reveal that the diagnosis submitted by the biospecimen collection site does not agree with the diagnosis rendered by the biobank. In 2010, the National Cancer Institute (NCI) organized a workshop entitled "Release of Research Results to Participants in Biospecimen Studies," which included a session devoted to such differences in diagnosis discovered in the course of biobanking. ${ }^{5}$ This situation is referred to as a "diagnostic discrepancy" and was defined agreement, an impartial and knowledgeable third party is consulted. The decision as to whether and how to notify research participants of any confirmed changes in diagnosis would be determined by institutional procedures. Implementation of this proposed process will require clear delineation of the roles and responsibilities of all involved parties in order to promote excellence in patient care and ensure that researchers have access to biospecimens of requisite quality.

Genet Med 2012:14(4):417-423

Key Words: biobank; biospecimen; diagnostic discrepancy; incidental finding; return of research results

by NCI workshop attendees as any diagnosis made on the biospecimen that is different from the clinical diagnosis, that is, the diagnosis listed in the annotation or pathology report associated with the biospecimen as submitted by the biospecimen collection site. ${ }^{5}$ A purported diagnostic discrepancy, however, may often reflect a difference of opinion and does not necessarily imply that the initial diagnosis was incorrect. Additionally, the discrepancy may be due to clerical errors, such as mislabeling of a biospecimen or sampling error such that the biospecimen submitted to the biobank has a different cell type from the one reviewed by the pathologist at the collection site (Table 1). Although there is little published data about the rate of diagnostic discrepancies discovered in the course of biobanking, a study of surgical pathology cases at one institution found that second-opinion review led to the discovery of major diagnostic discrepancies in $2.3 \%$ of total cases and corresponding changes in clinical management for $1.2 \%$ of total cases. ${ }^{6}$ Another study has shown that the frequency of errors in cancer diagnosis are institution-dependent and may occur in up to $11.8 \%$ of all cytologic-histologic specimen pairs. ${ }^{7}$

In addition to diagnostic discrepancies, standard pathological review at the biobank may also yield incidental findings previously unobserved at the collection site. In the context of biobanking, a "pathological incidental finding" is considered to be any clinically relevant information about a biospecimen discovered during review by the biobank that was not noted at the biospecimen collection site. "Incidental" should not be construed to mean that the finding is unimportant or insignificant, but instead that the finding is simply unexpected given the

${ }^{1}$ Office of Biorepositories and Biospecimen Research, National Cancer Institute, National Institutes of Health, Bethesda, Maryland, USA; ${ }^{2}$ Division of Cancer Biology, National Cancer Institute, National Institutes of Health, Bethesda, Maryland, USA. Correspondence: Nicole C. Lockhart (lockhani@mail.nih.gov)

Submitted 7 October 2011; accepted 17 January 2012; advance online publication 16 February 2012. doi:10.1038/gim.2012.11 

Table 1 Examples of elements to verify prior to initiating
diagnostic discrepancy process

Element to verify

Possible cause of error

Biospecimen is from the correct Mislabeling/clerical error related to research participant biospecimen

Biospecimen is the correct

biospecimen type

Biospecimen is from the correct clinical event

Associated clinical data (including pathology report) are from the correct research participant

Associated clinical data (including pathology report) are current and from the correct clinical event

Biospecimen or associated slides were reviewed by the pathologist at the collection site

Mislabeling/clerical error related to biospecimen

Mislabeling/clerical error related to biospecimen

Mislabeling/clerical error related to data

Mislabeling/clerical error related to data, including failure to provide current pathology report if the original report was amended

Pathologist at the collection site did not review the biospecimen and/or a sampling error occurred

medical context of the patient. ${ }^{5}$ This definition of a pathological incidental finding is somewhat distinct from definitions that have been described elsewhere ${ }^{1}$ in that it makes no reference as to whether the finding is within or outside the aims of the research study. Pathological incidental findings may arise if the biospecimen was not reviewed at the collection site, such as if it was obtained from a purportedly "healthy" donor, or if the biospecimen type was exempt from pathological review under institutional policy. Alternatively, a pathological incidental finding could arise if the biobank performs additional testing as part of their internal review process. An example of a pathological incidental finding would be the detection of leukemia cells in a bone marrow sample from a patient who was not believed to have leukemia. ${ }^{5}$

\section{ISSUES UNIQUE TO DIAGNOSTIC DISCREPANCIES AND PATHOLOGICAL INCIDENTAL FINDINGS}

Diagnostic discrepancies and pathological incidental findings could be considered a subcategory of incidental findings. Such findings raise several unique issues as well as added responsibilities that may not be encountered with other types of research results or incidental findings. Of primary importance is the fact that diagnostic discrepancies and pathological incidental findings would generally have known and established clinical utility and may be clinically actionable. Because differences in opinion occur in clinical pathology, there are professional mores and standard practices as to how such information should be handled that can be adapted to biobanking.

Unlike many other types of research results, diagnostic discrepancies and pathological incidental findings, in general, would not be discovered by researchers. Such findings would most likely occur as part of the quality control review performed by the biobank, either when the biospecimen is received or prior to the biospecimen being released to a researcher. Accordingly, the possibility of diagnostic discrepancies or pathological findings would not be tied to the research plan of the recipient investigator utilizing the biospecimens but would instead be based on the standard operating procedures and other policies of the biobank.

\section{Which biospecimens should be reviewed for discrepant diagnosis or pathological incidental findings?}

A key challenge for the biobank in this context is to define which categories of biospecimens should undergo routine pathological review. Participants in the NCI workshop noted that discrepant diagnoses or pathological incidental findings are most likely to occur on solid tissue biospecimens because such specimens are most likely to undergo diagnostic pathology review at the biospecimen collection site. ${ }^{5}$ Accordingly, NCI workshop participants recommended that "if a biospecimen is delivered to the biospecimen resource after or concurrent with a primary pathology clinical interpretation, a secondary pathology quality assurance evaluation should be performed, using standard best practice assays (for example, hematoxylin-and-eosin stain or cytology preparation), prior to banking and within a clinically reasonable period of time." Furthermore, NCI workshop participants recommended that for solid tissue biospecimens on which no primary histopathological interpretation was performed (e.g., "normal" biospecimens or biospecimen types exempt from pathological review by institutional policy), a pathology intake evaluation should be performed prior to biobanking and within a clinically reasonable period of time. ${ }^{5}$ Biobanks should consider whether or not the patient population or disease being studied would lend itself to a greater incidence of diagnostic discrepancies or pathological incidental findings and design policies accordingly. In practice, biobanks may not always have sufficient funding and/or staff to perform the recommended pathology review at intake and may opt instead for review of biospecimens prior to release to researchers. From an ethical perspective, however, timely notification of a discrepant diagnosis or pathological incidental finding will provide the greatest degree of benefit to the research participant. Notifying the biospecimen collection site several years after collection of a suspected discrepant diagnosis or pathological incidental finding may not be as effective due to potential difficulties in locating the pathologist of record, the original clinical biospecimen, or the research participant.

In addition to diagnostic review, some biobanks routinely perform additional clinical tests on banked biospecimens. Such testing could range, for example, from infectious disease screening of blood biospecimens to immunohistochemical assays for estrogen or progesterone receptors in breast tumors. Repeating or performing additional clinical assays may yield either diagnostic discrepancies or pathological incidental findings, if similar testing was not performed at the biospecimen collection site. A question that arises in this context is whether the biobank has a duty to perform or repeat clinical tests-a purported duty to hunt for possible discrepancies or pathological incidental findings. ${ }^{89}$ Given that the primary purpose of the biobank is to support research, imposing this type of responsibility would greatly increase cost, reduce the amount of resources available 
for research, and introduce possible medical and legal consequences for the biobank. Accordingly, NCI workshop participants recommended that biobanks should not be required to repeat clinical tests. ${ }^{5}$ However, if the biobank chooses to do so for scientific purposes and the results do not concur with those at the collection site, the results should be handled as a discrepancy or pathological incidental finding and reported as described below. ${ }^{5}$

\section{Should discrepant diagnoses or pathological incidental findings discovered in the course of biobanking be returned?} Although the specific situation of diagnostic discrepancies and pathological incidental findings has not generally been discussed in the ethics literature, many of the principles and criteria that have been developed for whether to return incidental findings and/or individual research results can be instructive. The National Bioethics Advisory Committee recommended that disclosure of research results to individuals be considered an "exceptional circumstance" that should only occur if the findings are scientifically valid and confirmed, the findings have significant implications for the subject's health concerns, and a course of action to ameliorate or treat these concerns is readily available. ${ }^{10}$ Wolf et al. ${ }^{1}$ recommended that incidental findings should be disclosed to research participants when such disclosure would constitute a "strong net benefit," such as "information revealing a condition likely to be life-threatening" or "information revealing a condition likely to be grave that can be avoided or ameliorated." The National Heart, Lung, and Blood Institute Working Group guidelines recommend that individual genetic research results be offered to research participants if all of the following criteria are met: the finding has important health implications for the participant with established and substantial associated risks, the finding is actionable, the finding is analytically valid, and the participant consented to receive his or her individual genetic results. ${ }^{2}$ Similarly, a working group focused on genomic research recommends that researchers involved in a biobank system should return incidental findings and individual research results if all of the following criteria are met: findings are analytically valid, return comports with applicable law (including the Clinical Laboratory Improvement Amendments), ${ }_{11}^{11}$ the research participant opted to receive findings, the findings reveal established and substantial risk of a serious health condition, and the findings are clinically actionable. ${ }^{3}$ The NCI workshop recommended that individual research results be considered for return if the result is analytically valid, clinically serious or significant, and clinically actionable and the research participant consented to the receipt of such information. ${ }^{5}$

The above recommendations and others highlight the common themes of analytical validity, importance to health, clinical actionability, and consent to receipt of the information as required for return of individual research results and/or incidental findings. Biobanks should aim to meet the standard of analytical validity through the use of best practice assays performed by board-certified pathologists. ${ }^{5}$ In some cases, biobanks may consider additional measures such as accreditation by the College of American Pathologists ${ }^{12}$ or the American Association of Tissue Banks ${ }^{13}$ or certification under the Clinical Laboratory Improvement Amendments. ${ }^{11}$ Biobanks may also consider adhering to voluntary recommendations such as the NCI Best Practices for Biospecimen Resources ${ }^{14}$ or the International Society for Biological and Environmental Repositories Best Practices for Repositories ${ }^{15}$ in order to ensure that their operations are consistent with industry standards. Determining whether a specific discrepancy is important to the research participant's health and clinically actionable will be dependent on the specifics of the case, but in general, one would expect most diagnostic discrepancies to meet this standard. Even if the likely change in diagnosis would not alter the care or treatment of the research participant at the time of discovery, it would still be important to appropriately report the discrepancy to ensure the accuracy of the research participant's medical record for future health-care decisions. Pathological incidental findings, however, may be either minor or major depending on the nature of the finding and may not always be considered important to the person's health. The question of whether a research participant must consent in order to receive information related to a diagnostic discrepancy or pathological incidental finding is difficult to resolve. In general, most research participants would probably expect that basic information vital to their health, such as their proper diagnosis, would be disclosed to them. However, biobanks generally do not discuss the issue of diagnostic discrepancy or pathological incidental findings within the informed consent document, and inclusion of such information is not explicitly recommended in regulations or guidance or best practice documents. The issue of how diagnostic discrepancies and pathological incidental findings should be addressed in informed consent documents is discussed further below.

\section{How should discrepant diagnoses and pathological incidental findings be returned?}

In addition to the question of whether biobanks should report diagnostic discrepancies and pathological incidental findings, there are also operational issues concerning the process for returning such findings. Pathologists in clinical practice frequently seek second opinions from colleagues pertaining to a particular diagnosis. ${ }^{5}$ With the clinical workflow in mind, participants in the NCI workshop devised a general flow chart (Figure 1) to illustrate how biobanks can address diagnostic discrepancies or pathological incidental findings. ${ }^{5}$ The proposed process includes the initial step of verifying that the proper biospecimen was provided to the biobank to ensure that the discrepancy is not the result of mislabeling, data entry errors, or other similar issues ${ }^{5}$ (Table 1). Once the identity of the biospecimen is confirmed, the diagnosis or finding is verified internally within the biobank via peer review. ${ }^{5}$ If the diagnostic discrepancy or pathological incidental finding persists, the pathologist at the biobank would contact the pathologist of record at the collection site. ${ }^{5}$ In some cases, the pathologist at the biobank 
may consider copying the pathology department chair and/or the principal investigator of the research study (if applicable) on communications to ensure that notification is received. The biobank should be prepared to supply pathological images or in

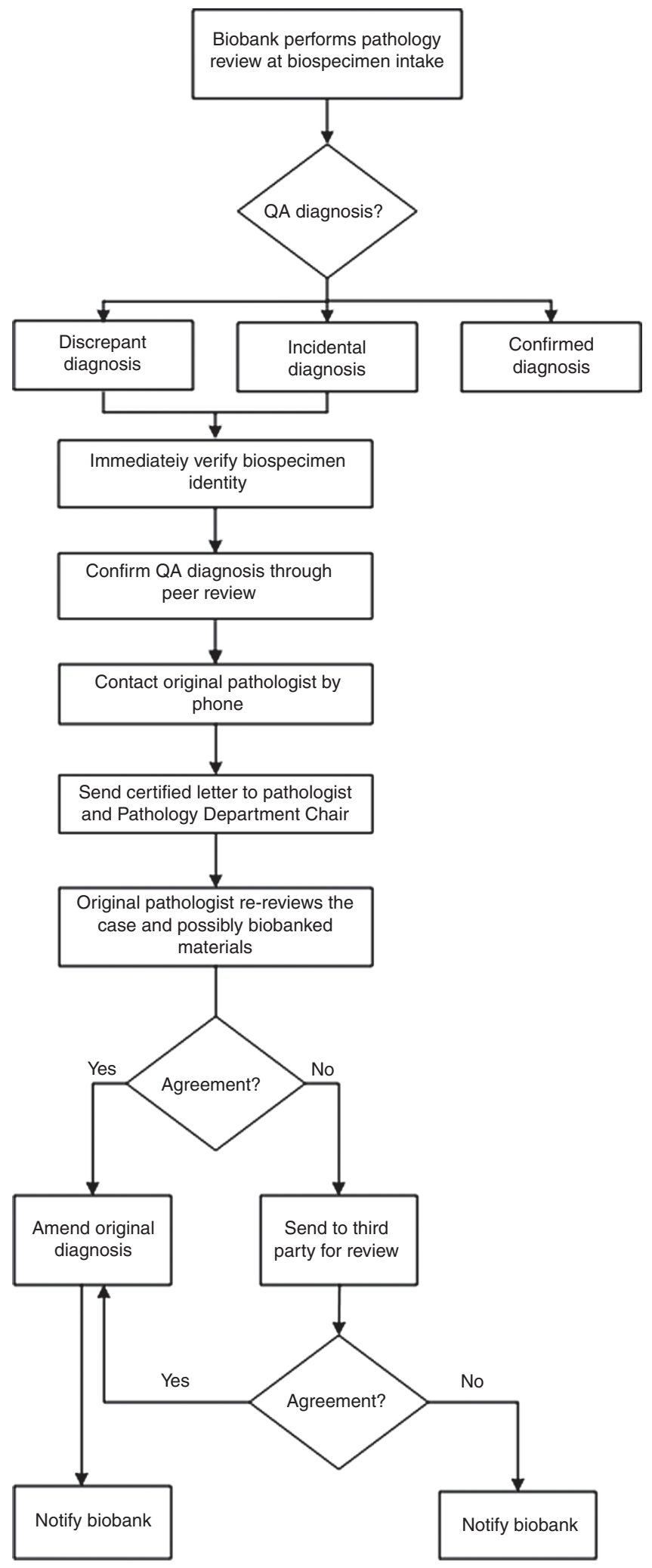

some cases the banked biospecimen if the pathologist of record at the collecting institution needs these materials to properly review the case..$^{5}$ If the pathologist at the biobank and the original pathologist do not reach agreement, an impartial and knowledgeable third party should be consulted. ${ }^{5}$ If that third party agrees with the pathologist at the biobank, the original diagnosis should be amended and the patient notified as appropriate using existing institutional procedures. ${ }^{5}$ If no agreement is reached, the biobank should be notified so that a decision may be made about future storage and use of the biospecimen. ${ }^{5}$

Consideration must also be given to whom the appropriate party is to communicate the finding to the research participant. The NCI workshop recommended that both discrepant diagnoses and pathological incidental findings be communicated from the biobank to the collection institution, preferably to the pathologist of record. ${ }^{5}$ Institutional processes would then be followed to communicate the finding to the research participant, if necessary. ${ }^{5}$ Some might deem the return of diagnostic discrepancies or pathological incidental findings to the treating physician and/or pathologist of record paternalistic ${ }^{16}$; however, it should be remembered that the majority of biospecimens originate from the clinical care system and the biobank may not have complete or current information on the research participant or a fiduciary relationship with him or her. Due to the significant risk of providing inaccurate or incorrect information, the biobank should never directly contact the research participant about a possible discrepancy or pathological incidental finding. If a pathological incidental finding is discovered in a biospecimen for which there is no preexisting pathology report, the biobank may choose to contact the participant's physician, if available, or the principal investigator of the research study at the collection site, who is in a better position to offer follow-up information. Discrepant diagnoses or pathological incidental findings should be communicated to the research participant by a knowledgeable person at the biospecimen collection site who should be prepared to offer clinical referrals if requested. Ideally, communication should be provided through a direct conversation with the participant and followed up by written documentation that could be shared with other health-care professionals if needed. ${ }^{3,5}$

Figure 1 Proposed process for handling diagnostic discrepancies and pathological incidental findings discovered in the course of review within the biobank. The proposed process includes verification that the proper biospecimen was provided to the biobank and verification of the diagnosis internally within the biobank. The pathologist at the biobank contacts the pathologist at the collection site via both phone and certified letter. The pathologist at the biospecimen collection site reviews the diagnosis, possibly including the banked biospecimen if necessary. If the pathologist at the biobank and the original pathologist do not reach agreement, an impartial and knowledgeable third party is consulted. If that third party agrees with the pathologist at the biobank, the original diagnosis is amended and the patient notified as appropriate using existing institutional procedures. If no agreement is reached, the biobank is notified. This figure is based on a proposed process developed at the $\mathrm{NCI}$ workshop on Release of Research Results to Participants in Biospecimen Studies. ${ }^{5} \mathrm{NCl}$, National Cancer Institute; QA, quality assurance. 
An additional reason to return diagnostic discrepancies or pathological incidental findings to the collection site is that the biobank generally will not have access to the research participant's identity or contact information. In most cases, a biospecimen is identified by a common identification number or code that is shared by the biobank and the collection site and linked to an individual research participant. The code enables communication about the biospecimen and protects the privacy of the research participant. Because many biobanks are not authorized to receive identifiable information, the code should not contain any protected health information or allow for linkage to the individual research participant. In studies that anticipate the discovery and return of diagnostic discrepancies or pathological incidental findings, a clear chain of custody must be established in order to ensure that the discrepancy or finding is associated with the correct research participant.

\section{IMPLEMENTATION CHALLENGES Operational considerations}

The details of how to implement the process outlined in Figure 1 will depend on the specific organizational model used by the biobank, as described in Figure 2. In model A, the collection site and the biobank reside within the same institution. This model presents fewer challenges related to implementation of these recommendations because many of the policies would apply to all entities. In model B, the entity serving as the biobank is external to the institution collecting the biospecimens, such as if the biobank is a contracted entity providing processing and storage services. Finally, model $\mathrm{C}$ depicts a biobanking network where multiple collection sites contribute biospecimens to a centralized biobank. In model $\mathrm{C}$, the components of the network may or may not be bound by contract or other binding agreements that govern the relationships between parties. Although small differences in the process used for return may be appropriate, entities involved in a network should harmonize criteria used to determine whether and how a finding will be reported to the collection site in order to promote equality across participating sites.

In addition to the organizational model, the implementation of these recommendations may be affected by several other factors. For example, the nature of the collection process and whether it is prospective or retrospective will impact the feasibility of implementation because biospecimens in the latter category may not have been reviewed at intake. Implementation may be more practicable for biobanks involved in clinical trials systems or otherwise closely integrated with clinical care, in which there may be existing mechanisms for review of biospecimens and established procedures for maintaining chain of custody. Finally, implementation can depend on the types of biospecimens stored and the overall purpose of the biobank. Population biobanks storing only blood or blood products may generally have a much lower incidence of diagnostic discrepancies or pathological incidental findings, whereas a higher rate of such findings may be observed by tissue biobanks.
Model A

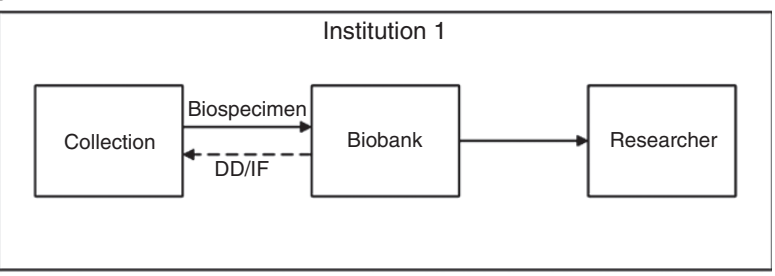

Model B

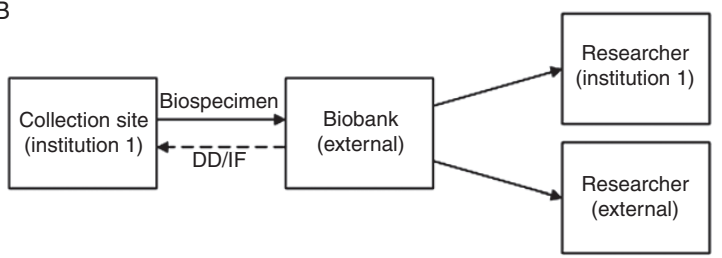

Model C

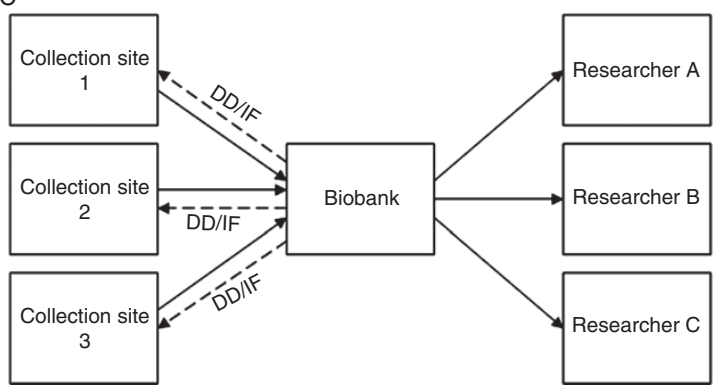

Figure 2 Common biobanking models. In model $A$, the collection site and the biobank reside within the same institution. In model B, the biobank is external to the institution collecting the biospecimens, but researchers may be internal or external to the collecting institution. In model C, multiple collection sites contribute biospecimens to a centralized biobank, the biobanking network, which then distributes biospecimens to researchers. Solid arrows represent transfer of biospecimens, and dashed arrows represent DDs/IFs. DD, diagnostic discrepancy; IF, incidental finding.

\section{Informed consent}

Although it has been recommended that research participants should provide consent prior to disclosure of individual research results or incidental findings, it is not clear whether the same standard could be applied to diagnostic discrepancies and pathological incidental findings. ${ }^{1-3,5} \mathrm{~A}$ group convened to provide guidance on ethical and legal issues to the NCI's Cancer Human Biobank (caHUB) recommended that the informed consent document notify individuals that their samples will be reviewed by the biobank and that any clinically relevant information regarding diagnosis will be communicated to them via their institution. ${ }^{17}$ Similarly, the NCI workshop recommended that "informed consent documents should include a general statement as to whether clinically significant differences of opinion pertaining to the diagnosis or other information related to the patient's health will be returned." ${ }^{\prime 5}$ Although this type of disclosure in the consent appears reasonable, care must be taken to avoid the perception by research participants that this additional examination of their tissue is a possible benefit or that their diagnosis is correct purely because they have not been contacted by the biobank. The issue of diagnostic discrepancy and pathological incidental findings should be addressed carefully in the informed consent document in order to clarify that participants will not be receiving an automatic "second opinion" 
if they consent to the study. There is a related issue of whether research participants should be offered the opportunity to opt out of receiving diagnostic discrepancies or pathological incidental findings. ${ }^{1,2,5}$ However, in the case of diagnostic discrepancies or major pathological incidental findings, the findings may be of such clinical importance that the biobank may feel that a "duty to rescue" is invoked ${ }^{18}$ and compelled to inform the collection site of the finding regardless of the research participant's desire for the information. Ultimately, the decision of whether to inform the research participant will be borne by the collection site, according to established institutional policies. Finally, as with all research results, diagnostic discrepancies and pathological incidental findings can only be disclosed if a link to the participant's identity is maintained. If biospecimens will be completely anonymized and return of diagnostic discrepancies or pathological incidental findings will not be possible, this should be addressed in the consent document.

\section{Economic factors}

Changes to biobanking practices, such as pathological review at biospecimen intake, will certainly require additional upfront investment. However, it must be remembered that failure to perform such quality-control procedures could result in a significant cost not only to the biobank but the research enterprise as a whole. If biospecimens are not reviewed at intake, the biobank may simply be deferring the cost of biospecimen review to a later date while paying to store biospecimens that will eventually fail to meet requisite quality standards. Biospecimen storage is considered one of the five key factors in biobanking cost models. ${ }^{19}$ There is also the more intangible cost to research progress when poor-quality biospecimens lead to either questionable research findings or to researchers limiting the scope of their research. A survey of cancer researchers found that over $50 \%$ of respondents sometimes or often questioned their work because of biospecimen quality concerns and over $40 \%$ of respondents often or usually limited their work because of difficulty in obtaining biospecimens that meet their needs. ${ }^{20}$ Increasing standards such as pathological review of biospecimens may require more resources in the short term, but such standards are necessary to meet the needs of research participants and researchers.

\section{CONCLUSION}

Diagnostic discrepancies and pathological incidental findings detected during the course of biobanking present unique challenges distinct from other types of research results or incidental findings. Given the potentially significant impact on patient care, diagnostic discrepancies should be returned to the pathologist of record and/or other designated staff at the biospecimen collection site. Although the basic process for return of diagnostic discrepancies may be based on standard clinical and institutional practices, the complexity and variety of biobanking models requires clear delineation of the roles and responsibilities of all involved parties. Ensuring that biospecimens are appropriately reviewed at intake to the biobank will promote researcher access to biospecimens of requisite quality and further advance biospecimen-based research.

\section{ACKNOWLEDGMENTS}

We thank all who participated in discussions at the National Cancer Institute (NCI)-sponsored workshop on Release of Research Results to Participants in Biospecimen Studies, particularly participants in the diagnostic discrepancy session. The diagnostic discrepancy session was chaired by Jared Schwartz and cochaired by N.C.L. Participants in the diagnostic discrepancy session included Joy Boyer, Lynn Dressler, Symma Finn, Marianne Henderson, Jennifer Hunt, Jennifer Loud, Mary Majumder, Mary Lou Smith, and Sheila Cohen Zimmet. Andrea Kelly of Rose Li and associates prepared the meeting summary. The meeting was held 8-9 July 2010, and the complete meeting summary and attendee list may be found at http://biospecimens.cancer.gov/resources/publications/ workshop/rrra.asp. This article was prepared for this symposium as part of work on National Institutes of Health (NIH)/National Human Genome Research Institute grant no. 2-R01-HG003178 on "Managing Incidental Findings and Research Results in Genomic Biobanks \& Archives" (S. Wolf, principal investigator), although the authors did not receive direct $\mathrm{NIH}$ funding for the preparation of this article. The opinions expressed in this article are the authors' own. The contents of this publication should not be construed to reflect the views or policies of the Department of Health and Human Services, the $\mathrm{NIH}$, or the $\mathrm{NCl}$. The work was supported by general appropriations to the $\mathrm{NCl}$.

\section{DISCLOSURE}

The authors declare no conflict of interest.

\section{REFERENCES}

1. Wolf SM, Lawrenz FP, Nelson CA, et al. Managing incidental findings in human subjects research: analysis and recommendations. J Law Med Ethics 2008;36:219-48, 211.

2. Fabsitz RR, McGuire A, Sharp RR, et al.; National Heart, Lung, and Blood Institute working group. Ethical and practical guidelines for reporting genetic research results to study participants: updated guidelines from a National Heart, Lung, and Blood Institute working group. Circ Cardiovasc Genet 2010;3:574-580.

3. Wolf SM, Crock BN, Van Ness, B et al. Managing incidental findings \& research results in genomic research involving biobanks \& archived datasets. Genetics in Medicine, 2012;14:361-384.

4. Clayton EW. Incidental findings in genetics research using archived DNA. J Law Med Ethics 2008;36:286-91, 212.

5. National Cancer Institute. Workshop on Release of Research Results to Participants in Biospecimen Studies. Bethesda, MD July 8-9 2010. http:// biospecimens.cancer.gov/resources/publications/workshop/rrra.asp. Accessed 5 August 2011.

6. Manion E, Cohen MB, Weydert J. Mandatory second opinion in surgical pathology referral material: clinical consequences of major disagreements. Am J Surg Pathol 2008;32:732-737.

7. Raab SS, Grzybicki DM, Janosky JE, et al. Clinical impact and frequency of anatomic pathology errors in cancer diagnoses. Cancer 2005; 104:2205-2213.

8. Richardson HS. Incidental findings and ancillary-care obligations. J Law Med Ethics 2008;36:256-70, 211.

9. Wolf SM, Paradise J, Caga-anan C. The law of incidental findings in human subjects research: establishing researchers' duties. J Law Med Ethics 2008;36:361-83, 214.

10. National Bioethics Advisory Commission. Research Involving Human Biological Materials: Ethical Issues and Policy Guidance, Volume I: Report and Recommendations of the National Bioethics Advisory Commission. 
Rockville, Maryland, August 1999. http://bioethics.georgetown.edu/nbad hbm.pdf. Accessed 5 August 2011.

11. Clinical Laboratory Improvement Amendments of 1988. Pub. L. No. 100578, 102 Stat. 2903

12. College of American Pathologists. Accreditation and Laboratory Improvement. http://www.cap.org/accreditation. Accessed 5 August 2011.

13. American Association of Tissue Banks. Accreditation Process. http://www. aatb.org/Accreditation. Accessed 5 August 2011.

14. National Cancer Institute. National Cancer Institute Best Practices for Biospecimen Resources. http://biospecimens.cancer.gov/bestpractices/. Accessed 5 August 2011.

15. International Society for Biological and Environmental Repositories 2008 Best Practices for Repositories: Collection, Storage, Retrieval and Distribution of Biological Materials for Research. Cell Preservation
Technology 2008;6(1). http://www.isber.org/bp/BestPractices2008.pdf Accessed 5 August 2011.

16. Fernandez C. Public expectations for return of results-time to stop being paternalistic? Am J Bioeth 2008;8:46-48.

17. National Cancer Institute. Preliminary Ethical, Legal and Social Considerations for the cancer Human Biobank (caHUB). June 2010.

18. Beskow LM, Burke W. Offering individual genetic research results: context matters. Sci Trans/ Med 2010;2:38cm20.

19. Vaught J, Rogers J, Carolin T, Compton C. Biobankonomics: developing a sustainable business model approach for the formation of a human tissue biobank. J Natl Cancer Inst Monographs 2011;2011:24-31.

20. Massett $\mathrm{HA}$, Atkinson NL, Weber $\mathrm{D}$, et al. Assessing the need for a standardized cancer HUman Biobank (caHUB): findings from a national survey with cancer researchers. I Natl Cancer Inst Monographs 2011;2011:8-15. 